\section{Adaptação e aplicabilidade do componente "maus-tratos" à estratégia da Atenção Integrada às Doenças Prevalentes na Infância no Brasil}

\section{Adaptation and applicability of the "mistreatment" component in Integrated Management of Childhood IIIness in Brazil}

\begin{abstract}
Objectives: to describe the process of adaptation to the Brazilian context and the applicability of the "mistreatment" module in the Integrated Management of Childhood Illness (IMCI) strategy, based on the Pan American Health Organization (PAHO) proposal.

Methods: the original protocol was translated into Portuguese, back-translated and reviewed by an independent observer. Features relating to legislation, the health context and the way Brazilian services are organized were incorporated. The materials were discussed by specialists from various areas until consensus was achieved with regard to the comprehensibility of the text and the correspondence between the wording and the intentions. The preliminary version was tested with a group of IMCI strategy monitors and suggestions arising from this were incorporated into the text. The final module was successfully applied during training of IMCI monitors in the Northeast region of the country.

Results: the material was found to be useful, clear and coherent. The ranking of degrees of severity of psychological mistreatment and negligence and texts providing guidelines for health workers and parents on the normal psychomotor and emotional development of children were included.

Conclusions: the incorporation of this module on mistreatment in the IMCI strategy's formal training sessions may fill a gap in the education of primary care health workers, who encounter problems relating to violence against children on a regular basis.
\end{abstract}

Key words Child abuse, Domestic violence, Integrated Management of Childhood Illness, Guideline Adherence
Sílvia Reis dos Santos 1

Ana Lúcia Ferreira 2

Antonio Carvalho da Paixão 3

Luci Yara Pfeiffer 4

Leda Amar Aquino 5

João Joaquim Freitas do Amaral 6

1 Instituto de Puericultura e Pediatria Martagão Gesteira. Universidade Federal do Rio de Janeiro. Av. Bruno Lobo 50, Ilha do Fundão. Rio de Janeiro, RJ, Brasil. CEP: 21.941-590

E-mail: sreisdossantos@gmail.com

2 Departamento de Pediatria. Faculdade de Medicina. Universidade Federal do Rio de Janeiro. Rio de Janeiro, RJ, Brasil.

3 Departamento de Medicina. Universidade Federal de Sergipe. Aracaju, SE, Brasil.

4 Secretaria Municipal de Saúde de Curitiba. Curitiba, PR, Brasil.

5 Secretaria Municipal de Saúde do Rio de Janeiro. Rio de Janeiro, RJ, Brasil.

6 Departamento de Saúde Materno Infantil. Faculdade de Medicina. Universidade Federal do Ceará. Fortaleza, CE, Brasil.

\section{Resumo}

Objetivos: descrever o processo de adaptação ao contexto brasileiro e da aplicabilidade do conteúdo do módulo de maus-tratos no âmbito da estratégia Atenção Integrada às Doenças Prevalentes na Infância (AIDPI), a partir do original proposto pela Organização Pan-Americana da Saúde.

Métodos: o protocolo original foi traduzido para o português, retro-traduzido e revisado de forma independente. Foram incorporados aspectos relativos à legislação, contexto de saúde e organização dos serviços brasileiros. O material foi discutido por especialistas de diferentes áreas até obter consenso a respeito de compreensão e correspondência sobre os conceitos e os instrumentos propostos. A versão preliminar foi testada com grupo de monitores da estratégia AIDPI. Sugestões foram incorporadas ao texto. O módulo final foi aplicado com sucesso em treinamento para monitores em AIDPI na Região Nordeste.

Resultados: o material mostrou-se útil, claro e coerente. A classificação de gravidade para maus tratos psicológicos e negligência, além de textos com orientações aos profissionais e pais sobre o desenvolvimento psicomotor e emocional normais da criança foram incluidos.

Conclusões: a incorporação desse módulo de maus-tratos em treinamentos formais na estratégia AIDPI pode preencher uma lacuna na educação do profissional de saúde na atenção primária, onde problemas relacionados à violência contra a criança são frequentes.

Palavras-chave Maus-tratos infantis, Violência doméstica, Atenção Integral às Doenças Prevalentes na Infância, Fidelidade a Diretrizes 


\section{Introdução}

O artigo $19^{\circ}$ da Convenção das Nações Unidas sobre os Direitos da Criança determina o direito à proteção integral de todas as crianças contra qualquer forma de violência. ${ }^{1}$ No entanto, abusos fatais e não fatais continuam a ocorrer no mundo inteiro, e as estatísticas existentes ainda não refletem toda a magnitude do problema. Na América Latina e no Caribe, $85 \%$ das mortes por maus-tratos são classificadas erroneamente como acidentais ou indeterminadas e, para cada morte, estimam-se nove incapacitados, 71 crianças com lesões graves e inúmeras vítimas com sequelas psicológicas. ${ }^{2,3}$

Trata-se então de grave problema de saúde pública, e o setor saúde tem papel importante ao propor, para o manejo inicial, o uso de metodologias de eficácia estabelecida na abordagem de outros problemas de saúde da criança, como a estratégia da Atenção Integrada às Doenças Prevalentes na Infância (AIDPI).

Na década de 1990, a Organização Mundial da Saúde (OMS) e o Fundo das Nações Unidas para a Infância (UNICEF) desenvolveram a estratégia AIDPI, que integra as diferentes intervenções disponíveis para a prevenção e tratamento das doenças na infância, bem como as ações de promoção da saúde, com potenciais de flexibilidade capazes de se adequar às mais diversas situações epidemiológicas. ${ }^{4}$ Através da utilização de sinais e sintomas de gravidade que apresentam boa relação de sensibilidade e especificidade, além de sistematização adequada do atendimento à criança doente, a estratégia tem por objetivo reduzir as mortes, a frequência, a gravidade e as sequelas das doenças que mais acometem as crianças menores de cinco anos. Proporciona, assim, aos profissionais de saúde, uma ferramenta prática para garantir atenção integrada e de qualidade às crianças com as quais entram em contato, sem omitir nenhuma das etapas mais importantes de avaliação. A sequência de etapas para avaliar a presença dos sinais de doença está organizada de tal forma que primeiro são identificados os sinais de gravidade, passando em seguida à verificação da presença dos diferentes sinais de doenças mais frequentes na infância. Terminadas essas etapas, a sequência da AIDPI inclui a verificação sistemática de aspectos preventivos e a promoção da saúde. 5

A estratégia vem se expandindo ao longo dos anos e incorporando conteúdos adicionais, por exemplo afecções perinatais e asma, acompanhando as mudanças do perfil epidemiológico de cada região ou país. 6 A violência contra a criança é outro pro- blema que tem se destacado na demanda dos diversos países das Américas como aspecto adicional da atenção infantil a ser incorporado na estratégia AIDPI. Frente à importância do problema e em resposta à demanda expressa, em 2003 a Organização Pan-Americana da Saúde (OPAS) organizou uma oficina de trabalho internacional com especialistas na área, voltada para a incorporação de novo componente "Maus-tratos na Infância" na estratégia AIDPI. O Brasil estava representado por um dos autores deste trabalho. Como produto deste encontro, foi elaborado em espanhol um módulo preliminar sobre "Detecção e prevenção de maustratos na infância no contexto da estratégia AIDPI". Esse módulo foi informalmente distribuído pela OPAS a consultores internacionais, sem desdobramentos no Brasil naquele momento. Em 2004, o Instituto de Puericultura e Pediatria Martagão Gesteira, da Universidade Federal do Rio de Janeiro (IPPMG/UFRJ), submeteu à Área Técnica da Saúde da Criança do Ministério da Saúde do Brasil uma proposta de trabalho com o objetivo de traduzir para o português este novo protocolo clínico, adaptandoo à realidade brasileira. Essa proposta foi aprovada em 2005 e recebeu financiamento da Fundação Nacional de Saúde, sendo executada ao longo de 2006.

O presente trabalho tem como objetivo descrever o processo de adaptação ao contexto brasileiro e da aplicabilidade do conteúdo do módulo de maustratos no âmbito da estratégia AIDPI, a partir do original proposto pela Organização Pan-Americana da Saúde.

\section{Métodos}

O texto preliminar elaborado em 2003 foi inicialmente traduzido para o português por dois tradutores e retro-traduzido de forma independente, cujas versões foram comparadas e eventuais discordâncias foram discutidas e acordadas entre os tradutores. $\mathrm{O}$ material foi adaptado à realidade nacional, com a incorporação de textos e procedimentos previstos na legislação brasileira sobre o tema.

O material produzido foi apresentado e discutido em primeira oficina de revisão e adaptação, que contou com a presença de 11 profissionais de saúde de diversas regiões do país (médicos pediatras, ginecologista e epidemiologista com vasta experiência na área de violência doméstica, além de facilitadores "seniors" na estratégia AIDPI). Estes participantes foram selecionados por serem reconhecidamente especialistas em suas áreas de atuação: nas escolas médicas e hospitais universitários, nos comitês de 
defesa da criança de sociedades profissionais, em serviços públicos e organizações não governamentais de referência voltadas para a proteção e atenção aos maus-tratos na infância. As modificações e inserções inicialmente propostas por este grupo foram sistematizadas e novamente apresentadas e discutidas em outra oficina de trabalho, desta vez com a presença de seis dos 11 participantes iniciais. Os seis participantes passaram a constituir um grupo central, que se manteve ativo até o fim dos trabalhos e são os autores deste artigo. O produto dessa segunda reunião, embora ainda preliminar, foi considerado adequado para a etapa seguinte.

A fim de identificar termos locais para as perguntas que constavam na nova ficha de atendimento da criança, foram entrevistados profissionais de saúde e os responsáveis pelas crianças. Esta etapa foi previamente submetida e aprovada pelo Comitê de Ética em Pesquisa do Instituto de Puericultura e Pediatria Martagão Gesteira da Universidade Federal do Rio Janeiro (IPPMG/UFRJ) e todos os participantes tiveram garantia de sigilo e anonimato. O trabalho foi organizado de acordo com orientações da OPAS/OMS (guia para a adaptação de material de curso) 7 e, utilizando-se de modelo de questionário proposto e adaptado para o tema, foram entrevistados cinco responsáveis por crianças atendidas no Ambulatório Materno-Infantil e na Emergência do IPPMG/UFRJ e quatro profissionais (dois médicos e duas enfermeiras) desses setores. Os termos correntes identificados foram tabulados em planilha, analisados e incorporados ao questionário do módulo. As modificações foram novamente discutidas em nova oficina de trabalho com o grupo central de consultores e a versão quase-final do módulo foi elaborada.

Em seguida, este novo material (módulo, fichas e anexos) foi testado em campo. Para tal, foi realizado treinamento de 16 horas de duração, para 12 médicos pediatras de diversas instituições e regiões do país, todos facilitadores da estratégia AIDPI. O treinamento teórico, com a presença dos consultores/profissionais que participaram da elaboração do módulo, teve a duração de oito horas e constou de leitura do módulo adaptado e discussão em grupo. Logo após essa etapa, sob a supervisão dos mesmos consultores, os participantes foram divididos em grupos para o atendimento a crianças com a aplicação da Ficha de Atendimento em AIDPI adaptada especificamente para este treinamento, com a inclusão dos quadros de avaliação e classificação de maus-tratos. Esta etapa foi executada em três diferentes unidades de saúde de uma mesma área do
Município do Rio de Janeiro: hospital universitário pediátrico (emergência e ambulatório maternoinfantil), hospital municipal geral de emergência (pronto-atendimento) e hospital municipal pediátrico de nível secundário (pronto-atendimento do ambulatório de pediatria) Os participantes foram orientados a anotar, em formulário próprio, todas as dúvidas, incongruências e/ou dificuldades encontradas na compreensão e aplicação do módulo na prática.

A Ficha de Atendimento em AIDPI construída foi aplicada a 30 crianças com idades de dois meses a menos de cinco anos (dez em cada unidade) e, de acordo com a experiência clínica dos consultores, foram identificadas duas crianças suspeitas de maustratos. Os dois casos foram notificados ao Conselho Tutelar, seguindo a legislação vigente em nosso país (Estatuto da Criança e do Adolescente). 8 Os pequenos grupos e os consultores anotaram suas impressões sobre a aplicação na prática. As dificuldades, críticas e sugestões foram amplamente discutidas e analisadas em plenária, onde os participantes manifestaram com unanimidade a pertinência da iniciativa, além da necessidade e adequação da incorporação do módulo de maus-tratos à estratégia AIDPI.

De acordo com os resultados dessa experiência, o grupo central de consultores sistematizou a análise realizada, discutiu suas próprias impressões a respeito da aplicação da ficha e fez as alterações no módulo e na ficha da estratégia, procurando superar as dúvidas e dificuldades apontadas. Em fins de 2006 o módulo foi apresentado ao Ministério da Saúde do Brasil e divulgado em evento nacional organizado pela Área Técnica de Saúde da Criança e Aleitamento Materno do Ministério da Saúde.

Posteriormente, o material foi utilizado para treinamento de médicos e enfermeiros facilitadores da estratégia AIDPI na cidade de Teresina, na Região Nordeste do país, por solicitação da Fundação Municipal de Saúde local. A única modificação feita à época foi a substituição da ficha de notificação pelo novo modelo proposto pelo Ministério da Saúde (Ficha de investigação individual de violência doméstica, sexual e/ou outras violências), que estava sendo implantada no município naquela ocasião.

\section{Resultados}

O módulo, na íntegra, pode ser acessado no seguinte endereço eletrônico: http://www.ippmg.org.br/pdf/ AIDPI.pdf. A Tabela 1 corresponde à Avaliação e a Tabela 2 à Classificação dos maus-tratos. 
Avaliação dos maus-tratos (a ser incluído na Ficha de Avaliação da criança de dois meses a menos de cinco anos de idade - Estratégia de Atenção Integrada às Doenças Prevalentes da Infância.

Observar se:

- A criança expressa espontaneamente que sofre maus-tratos.

- $O(a)$ acompanhante expressa espontaneamente que a criança é vítima de maus tratos.

- Há evidência de alteração no comportamento da criança: agressiva, muito assustada, retraída, apática, evita o contato visual e/ou físico, apresenta condutas destrutivas ou autodestrutivas.

- Há evidência de alteração no comportamento dos pais ou cuidadores: indiferentes, descuidados, intolerantes, castigadores, que têm atitudes depreciativas, agressivas ou na defensiva.

Verificar se há:

- Lesões físicas sugestivas de abuso físico:

Lesões bilaterais, simétricas, em diferentes estágios de cicatrização, em áreas cobertas do corpo ou em regiões posteriores, dorso das mãos, costas e glúteos, áreas internas das coxas, mamas e genitais.

- Lesões físicas sugestivas de abuso sexual:

$\mathrm{Na}$ área genital e perianal: corrimento, secreção, sangramento, lesões (lacerações, úlceras, verrugas, hematomas, equimoses, etc.) ou cicatrizes.

- Lesões físicas inespecíficas:

Lesões de pele localizadas em áreas expostas, em geral são poucas e não têm forma limitada, nem um padrão definido; na área genital a presença de hiperemia por monilíase ou dermatite das fraldas; arranhaduras por prurido devido à infestação por oxiurus e escabiose; e fissura anal devido à constipação.

- Alterações sugestivas de negligência:

Criança mal-cuidada em sua higiene (asseio, dentes, cabelo, unhas); discrepância entre o aspecto do cuidador e da criança.

Criança mal-cuidada em sua saúde: consulta é tardia para a causa ou esquema de vacinação está incompleto ou criança se encontra desnutrida ou com atraso no desenvolvimento psicomotor.

\section{- Havendo qualquer lesão física ou fratura}

Perguntar:

Como se produziram os machucados/as feridas/os ferimentos?

Quando se produziram os machucados/as feridas/os ferimentos?

- Há história de acidentes/ traumatismos frequentes?

Determinar se:

Há discrepância entre as lesões e a história/ estágio de desenvolvimento

Para todos os responsáveis:

Perguntar:

- Como ensinam/educam/dão limites para este filho(a)?

- Como ensinam/educam/ disciplinam/dão limites aos outros filhos?

- Como resolvem os problemas/conflitos familiares?

Caso haja relato de agressões físicas ou psicológicas,

Determinar se:

Há ameaça à vida da criança

Perguntar:

Como o responsável justifica a agressão?

Nota: As diferenças em relação ao módulo original estão assinaladas em negrito. 
Classificação dos maus-tratos (a ser incluído na Ficha de Avaliação da criança de dois meses a menos de cinco anos de idade - Estratégia de Atenção Integrada às Doenças Prevalentes da Infância.

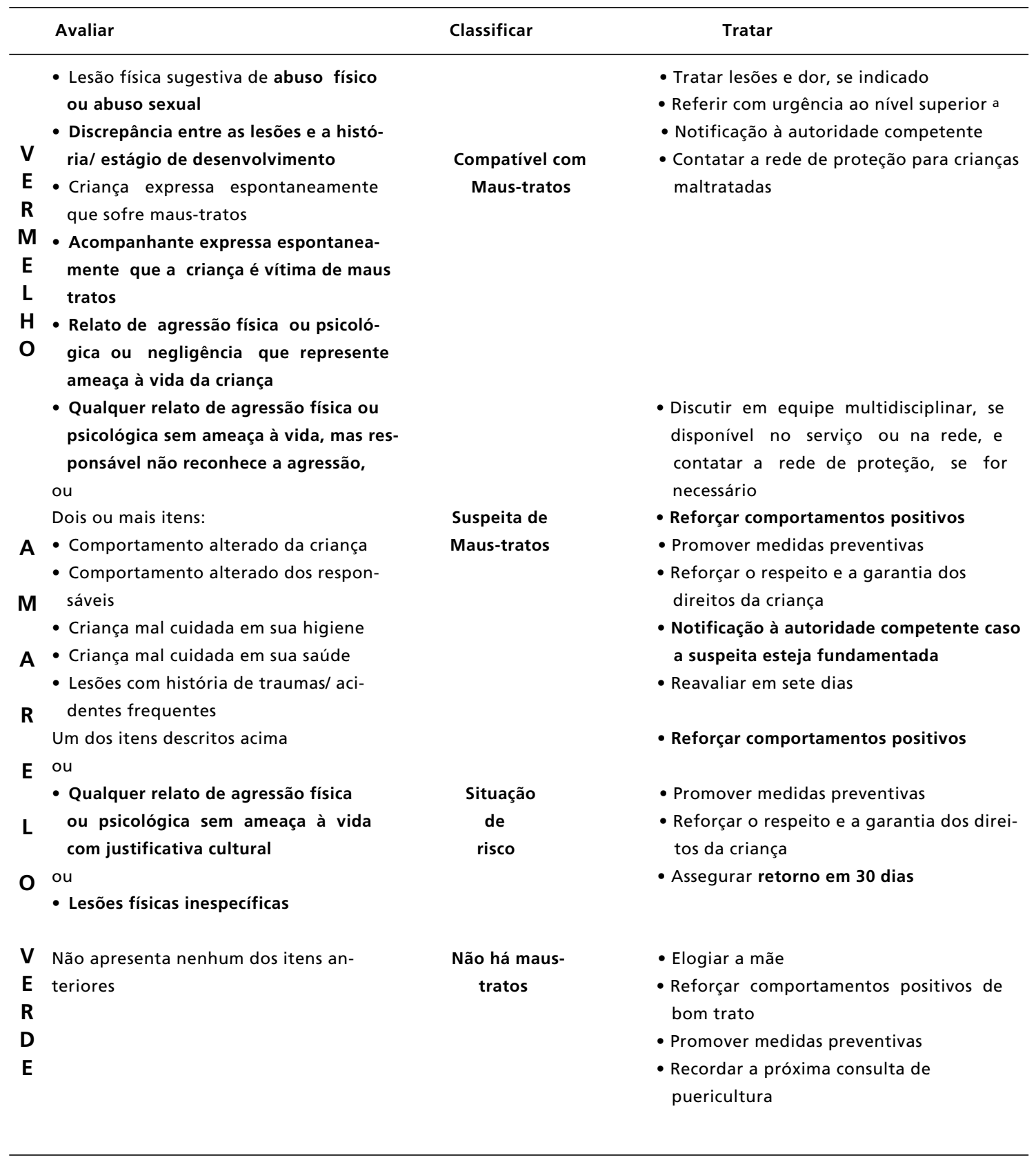


Avaliando a compreensibilidade das perguntas

A partir da avaliação preliminar da compreensibilidade das perguntas com profissionais da rede e com responsáveis, foram identificadas diferenças importantes de linguagem e sentido, sendo realizadas as seguintes alterações nas perguntas dirigidas aos responsáveis:

O termo "lesões" foi substituído por "machucados/feridas/ferimentos; "traumas frequentes" foi substituído por "acidentes/traumatismos frequentes" (a interpretação dada pelos entrevistados à palavra "trauma" era no sentido de trauma psíquico);

A pergunta "como disciplina seu filho" foi substituída por "como ensina / educa / dá limites para este filho", acrescentando-se a mesma pergunta para "outros filhos", uma vez que o profissional pode estar atendendo um lactente jovem cujos pais ainda não se utilizam de métodos educativos direcionados a ele próprio, mas podem utilizar a punição física com irmãos mais velhos, o que poderia indicar predisposição para, no futuro, repetirem a prática com a criança em questão;

A pergunta "como solucionam os conflitos em casa?" foi substituída por "como resolvem os problemas/conflitos familiares?".

\section{As inclusões}

Foram incluídos dados nacionais e um breve histórico sobre o problema dos maus tratos, ressaltando as consequências que trazem para as crianças, assim como conceitos e definições existentes em documentos da OMS2 e do Ministério da Saúde do Brasil. 9

No módulo original, as perguntas elaboradas para a detecção do uso de práticas de punição corporal pelos responsáveis não influenciavam na classificação final, apenas serviam para fomentar uma discussão com os pais a respeito de práticas disciplinares. Uma vez que a punição corporal está intimamente relacionada ao abuso físico ${ }^{10} \mathrm{e}$ agressões psicológicas também podem representar ameaça à vida da criança, ${ }^{11}$ optou-se por acrescentar outra pergunta: "Como o responsável justifica a agressão?", no caso de haver relato de agressões físicas ou psicológicas em resposta às primeiras questões, passando esta avaliação a interferir na classificação final.

A avaliação desse conjunto de respostas permitiu identificar situações novas para as classificações vermelha e amarela, descritas na Tabela 2, enquanto no material original havia quatro classificações: uma vermelha (compatível com maus-tratos físicos e/ou abuso sexual), uma amarela (suspeita de maustratos) e duas verdes (falha na criação e não há suspeita de maus-tratos).

\section{Os novos anexos}

Foram acrescentados quatro novos anexos ao material original: uma tabela com os marcos do desenvolvimento da criança até seis anos de idade, adaptado da Caderneta de Saúde da Criança; 11 a portaria do Ministério da Saúde que dispõe sobre a notificação de maus-tratos contra crianças e adolescentes atendidos nas entidades do Sistema Único de Saúde do país; 12 a ficha de notificação para casos suspeitos ou confirmados de maus-tratos, com o respectivo instrutivo; $13 \mathrm{e}$, por fim, um texto que orienta os pais sobre a educação dos filhos de forma não violenta. 14

Ao anexo já existente sobre legislação foram acrescentados artigos de interesse para o tema, contidos no Código Penal Brasileiro e no Estatuto da Criança e do Adolescente. 8,15

\section{As alterações no quadro de classificação}

A classificação vermelha, amarela e verde e respectivas condutas foram alteradas a partir das diversas discussões da equipe de consultores, com base no Relatório Mundial sobre Violência e Saúde² e em documentos da Sociedade Brasileira de Pediatria e de outras instituições e autores que estudam o tema. $3,16,17$

As diferenças entre a classificação do material original e a proposta a ser utilizada no Brasil encontram-se em destaque na Tabela 2.

A avaliação feita pelos participantes enfatizou os seguintes aspectos: o módulo é claro e coerente; as atividades práticas são fundamentais para a percepção de como se dá a abordagem aos maustratos; o treinamento vem suprir uma necessidade de embasamento teórico para problemas já enfrentados na assistência básica e em outros níveis de atenção.

\section{Discussão}

A dimensão do cuidado a ser prestado à criança aparentemente saudável tem sido repensada, pois a situação de saúde prevê o bem-estar físico, psíquico, social e moral do indivíduo. No entanto, muitas são as dificuldades para a inserção na prática clínica de temas que fogem à formação atual dos profissionais de saúde, em especial do médico. Estar apto a identificar o sofrimento infligido a uma criança, que vai além das doenças orgânicas, implica no saber dirigido ao particular, à especificidade de cada um e 
de cada história de desenvolvimento e relacionamento entre as gerações, com base numa reformulação da visão do poder familiar. Há que se priorizar a pesquisa de sinais das enfermidades físicas já reconhecidas como prevalentes, como também da violência, tão mortal ou produtora de danos como as primeiras. 18

É neste hiato da formação e atuação do profissional de saúde, sobretudo daquele que presta cuidados primários, que entendemos a pertinência e relevância da estratégia AIDPI e do módulo de maus-tratos acima apresentado. A estratégia AIDPI, em sua proposta inicial, não abordava especificamente os problemas precipitantes ressaltados no trabalho supracitado. No entanto, já possibilitava a detecção de maus-tratos através de outros indicadores como, por exemplo, o atraso de vacinas, o tempo excessivo de demora para a primeira consulta, o não comparecimento à consulta de retorno e a desnutrição, que podem representar negligência. Ao sugerir que se valorizem os atrasos nos marcos esperados para o desenvolvimento e ao acrescentar sinais mais específicos de maus-tratos, o módulo aqui apresentado permite que o profissional se aproxime também de outras formas de maus-tratos.

A inclusão do tema na estratégia AIDPI foi uma tarefa bastante árdua. Trata-se de um problema de caráter distinto dos demais já contemplados pela estratégia e sem sinais e sintomas específicos para direcionar as classificações. Acrescenta-se a esta dificuldade o fato de a violência ser um tema complexo, que requer uma abordagem delicada, uma vez que envolve questões e valores individuais, sociais e culturais, que são transmitidos de geração a geração, e que muitas vezes mascaram uma realidade incômoda: responsáveis podem maltratar e até levar à morte seus filhos. O processo de trabalho em grupo, presencial e à distância, com profissionais experientes de diversas áreas, foi intensivo, extensivo e desafiador. Diferentes conceitos, experiências e visões sobre o tema e sua inserção na estratégia AIDPI foram intensamente discutidos; impasses foram reconhecidos e enfrentados até que o consenso fosse possível. Ainda assim foram necessárias sucessivas aproximações até o esgotamento do tema, antes e após o piloto e o teste no campo. As alterações

\section{Referências}

1. Organização das Nações Unidas. Convenção sobre os direitos da criança. Assembléia Geral Resolução 44/25; 20 de novembro de 1989. [acesso em: 20 abr 2008]. Disponível em: http://www.onu-brasil.org.br/doc_crianca.php. propostas para o módulo foram baseadas no conhecimento e na experiência crítica dos profissionais e especialistas envolvidos na sua elaboração e entendemos que esta abordagem metodológica adotada foi fundamental para o sucesso da empreitada.

No treinamento de facilitadores, ficou patente a elevada frequência (não aferida) de uso de práticas de punição física para a "educação" das crianças, a facilidade e naturalidade com que o problema era relatado aos profissionais e, ao mesmo tempo, a receptividade das famílias para conversar a respeito e receber as orientações previstas. Dessa forma, ao incorporar perguntas relativas à educação dos filhos em todas as consultas, o novo módulo da estratégia AIDPI abre a possibilidade para uma conversa com os pais/responsáveis a este respeito, permitindo não apenas detectar desvios que possam levar ou já correspondam ao abuso físico, como também esclarecer e apresentar formas não violentas de educar. Constitui-se então em um instrumento útil para profissionais que lidam com famílias e se utilizam da estratégia AIDPI para avaliar as crianças. No entanto, ficou evidente que o ponto mais vulnerável da proposta diz respeito à dificuldade de referência para os casos indicados.

É importante que este material, ao mesmo tempo complexo pelo seu conteúdo e claro para sua aplicação, seja colocado à disposição de todos os profissionais de saúde e inserido nos treinamentos regulares na estratégia AIDPI. Para que as medidas de proteção às crianças de risco identificadas na aplicação do módulo sejam instauradas, faz-se necessário o desenvolvimento de um trabalho em rede intersetorial.

Uma vez que a estratégia AIDPI tem como proposta classificar problemas e não fazer diagnósticos, novos estudos epidemiológicos são necessários para verificar a acurácia da classificação "compatível com maus-tratos".

Cabe lembrar, por fim, que o módulo proposto deve seguir a legislação do país em relação às normas de notificação de violência e aos instrumentos utilizados para tal e, como estes últimos vêm recentemente sendo revistos e alterados, é importante a atualização desse item antes de sua aplicação.

2. Runyan D, Wattam C, Ikeda R, Hassan F, Ramiro L. Abuso infantil e negligência por parte dos pais e outros responsáveis. In: Krug EG, Dahlberg LL, Mercy JA, Zwi AB, Lozano R, editores. Relatório mundial sobre a violência e a 
saúde. Genebra: Organização Mundial da Saúde; 2002. p. 57-86.

3. Deslandes SF, Assis SG, Santos NC. Violência envolvendo crianças no Brasil - um plural estruturado e estruturante. In: Brasil. Ministério da Saúde. Impacto da violência na saúde dos brasileiros. Brasília, DF, Ministério da Saúde; 2005. p. 43-78.

4. Tulloch J. Integrated approach to child health in developing countries. Lancet. 1999; 354 (2 Supl): 16-20.

5. Gove S. Integrated management of childhood illness by outpatient health workers: technical basis and overview. The WHO Working Group on Guidelines for Integrated Management of the Sick Child. Bull World Health Organ. 1997; 75(1 Supl): 7-24.

6. OPAS (Organização Pan-Americana da Saúde), OMS (Organi-zação Mundial da Saúde). XXVI Conferência Sanitária Pan-Americana; 54 $4^{\mathrm{a}}$ Sessão do Comitê Regional. Washington, DC, EUA, 23-27 de setembro de 2002. [acesso em 06 jun 2009]. Disponível em http://www.paho.org/ portuguese/gov/csp/ csp26-4-p.pdf.

7. OPAS (Organización Panamericana de la Salud). Atención Integrada a las Enfermedades Prevalentes de la Infancia (AIEPI). Guia de adaptación para las políticas, normas clinicas, módulos y graficas clinicas para el curso OMS/UNICEF. Washington, DC; 1996. (OPS/HCT/ART/ CDD/96.24).

8. Brasil. Presidência : Casa Civil, Lei $8069 / 90$ de 13 de julho de 1990: dispõe sobre o Estatuto da Criança e do Adolescente. [acesso em: 6 de jun. 2009] 1990. Disponível em: www.planalto.gov.br/civil/leis/18069.html

9. Brasil. Ministério da Saúde. Violência contra a criança e o adolescente: proposta preliminar de prevenção e assistência à violência doméstica. Brasília, DF, 1997.

10. Kairys SW, Johson CF. Committee on Child Abuse and Neglect. The psychological maltreatment of children - technical report. Pediatrics. 2003; 109: e68.
11. Brasil. Ministério da Saúde. Caderneta de Saúde Brasil 2006. [acesso em: 24 abr. 2008] Disponível em: http://dtr2004.saude.gov.br/nutricao /documentos/caderneta_saude_da_crianca.pdf

12. Brasil. Ministério da Saúde. Portaria n. ${ }^{\circ}$ 1968/GM: Dispõe sobre notificação, às autoridades competentes, de casos de suspeita de confirmação de maus-tratos contra crianças e adolescentes atendidos nas entidades do sistema Único de saúde. Diário Oficial da União (DOU); Brasília, DF, (2006) em 26 out, 2001.

13. Brasil. Ministério da Saúde. Notificação de maus-tratos contra crianças e adolescentes pelos profissionais de saúde: um passo a mais na cidadania em Saúde. Brasília; 2002 (Série A, n. 167).

14. Pfeiffer L. Sugestão de abordagem aos pais sobre a importância dos vínculos. In: Campos JA, Paes CEN, Blank D, Costa DM, Pfeiffer L,Waksman RD, editores. Manual de segurança da criança e do adolescente. São Paulo: Sociedade Brasileira de Pediatria; 2004. p. 44-6

15. Brasil. Código Penal. [acesso em: 4 set. 2006]. Disponível em: http://www.bibvirt. futuro.usp.br / textos / humanas / legislacao/codpenal/codpenal.html. .

16. Pfeiffer L, Waksman R. Violência contra crianças e adolescentes. In: Campos JA, Paes CEN, Blank D, Costa DM, Pfeiffer L, Waksman RD, editores. Manual de segurança da criança e do adolescente. São Paulo: Sociedade, Brasileira de Pediatria; 2004. p. 195-267.

17. Sociedade Brasileira de Pediatria. Centro LatinoAmericano de Estudos de Violência e Saúde Jorge Carelli, Secretaria de Estado dos Direitos Humanos do Ministério da Justiça. Guia de atuação frente a maus-tratos na infância e na adolescência. Rio de Janeiro: 2001.

18. Schor EL. Rethinking well-child care. Pediatrics. 2004; 114: $210-6$.

Recebido em 29 de novembro de 2008

Versão final apresentada em 4 de junho de 2009

Aprovado em 6 de julho de 2009 